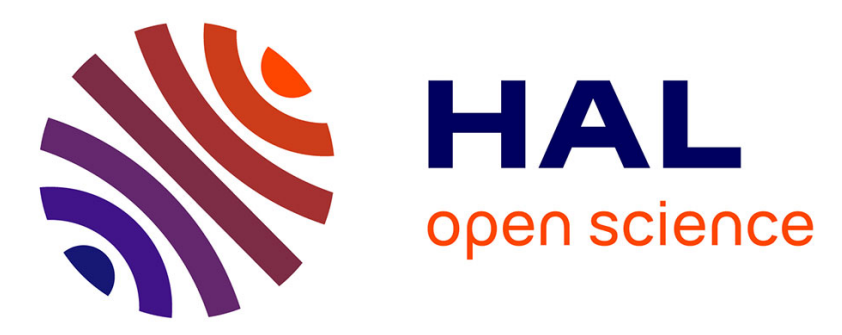

\title{
An Unified Approach for Blind Source Separation Using Sparsity and Decorrelation
}

Fangchen Feng, Matthieu Kowalski

\section{To cite this version:}

Fangchen Feng, Matthieu Kowalski. An Unified Approach for Blind Source Separation Using Sparsity and Decorrelation. 23rd European Signal Processing Conference (EUSIPCO 2015), Aug 2015, Nice, France. hal-01199620

\author{
HAL Id: hal-01199620 \\ https://hal.science/hal-01199620
}

Submitted on 15 Sep 2015

HAL is a multi-disciplinary open access archive for the deposit and dissemination of scientific research documents, whether they are published or not. The documents may come from teaching and research institutions in France or abroad, or from public or private research centers.
L'archive ouverte pluridisciplinaire HAL, est destinée au dépôt et à la diffusion de documents scientifiques de niveau recherche, publiés ou non, émanant des établissements d'enseignement et de recherche français ou étrangers, des laboratoires publics ou privés. 


\title{
AN UNIFIED APPROACH FOR BLIND SOURCE SEPARATION USING SPARSITY AND DECORRELATION
}

\author{
Fangchen Feng*, Matthieu Kowalski ${ }^{*, \dagger}$ \\ * Laboratoire des Signaux et Systèmes (Univ Paris-Sud, CNRS, CentraleSupélec) \\ Gif-sur-Yvette, France \\ ${ }^{\dagger}$ Parietal project-team, INRIA, CEA-Saclay, France
}

\begin{abstract}
Independent component analysis (ICA) has been a major tool for blind source separation (BSS). Both theoretical and practical evaluations showed that the hypothesis of independence suits well for audio signals. In the last few years, optimization approach based on sparsity has emerged as another efficient implement for BSS. This paper starts from introducing some new BSS methods that take advantages of both decorrelation (which is a direct consequence of independence) and sparsity using overcomplete Gabor representation. It is shown that the proposed methods work in both under-determined and overdetermined cases. Experimental results illustrate the good performances of these approaches for audio mixtures.
\end{abstract}

Index Terms - Blind Source Separation; Sparsity; Independant Component Analysis; Optimization

\section{INTRODUCTION}

The instantaneous linear mixture model of BSS assumes that:

$$
\mathbf{x}=\mathbf{A} \mathbf{s}+\mathbf{e}
$$

where $\mathbf{x} \in \mathbb{R}^{M \times T}$ and $\mathbf{s} \in \mathbb{R}^{N \times T}$ are the matrices of mixture channels and source signals respectively. $\mathbf{A} \in \mathbb{R}^{M \times N}$ is the mixing matrix and $\mathbf{e} \in \mathbb{R}^{M \times T}$ models the background noise.

The ICA [1] methods are often applied when $M \geq N$ (over-determined case). These methods try to achieve separation by minimizing an independence criterion between the components of the estimated sources. In the underdetermined case $(M<N)$, two-steps methods based on sparsity are largely used [2]: The mixing system is first estimated using clustering methods [3], then the sources are estimated thanks to optimization approaches [4].

On the other hand, it was shown in [5] that ICA methods (Infomax and FastICA) separate sparse sources rather than independent sources for fMRI (functional magnetic resonance imaging ) in certain cases. They conclued that one

This work benefited from the support of the "FMJH Program Gaspard Monge in optimization and operation research", and from the support to this program from EDF. should develop alternating decomposition methods targeting decompositions into sparse components rather than independent components. This idea greatly motivates this work and this article shows that by combining the decorrelation assumption, which is a direct consequence of independence, and sparsity, we can achieve better separation performances.

The contributions of this paper are twofolds: first, we offer a combination of the decorrelation assumption and the sparse model. This is the first time, to our knowledge, that such a combination is used for BSS. Second, we propose several efficient optimization algorithms mostly with proven convergence which work in both under-determined and overdetermined case.

The rest of this paper is organized as follow. Section 2 presents the convergence study of a typical separation method. We show our proposed methods in Section 3 and the experiments in Section 4.

\section{NOTATIONS AND STATE-OF-THE-ART}

\subsection{Notations}

Let us denote by $\boldsymbol{\Phi} \in \mathbb{C}^{T \times B}$ the matrix representing an energy-preserving STFT (Short-Time Fourier Transform) operator (or Parseval Gabor frame), the sources s can be resynthesized from their synthesis coefficients $\boldsymbol{\alpha} \in \mathbb{C}^{N \times B}$ by $\mathbf{s}=$ $\boldsymbol{\alpha} \boldsymbol{\Phi}^{*}$, where $\boldsymbol{\Phi}^{*} \in \mathbb{C}^{B \times T}$ is the adjoint operator of $\boldsymbol{\Phi}$, that is its Hermitian transpose. We propose to formulate the BSS problem as an optimization problem:

$$
\min _{\boldsymbol{\alpha}, \mathbf{A}} f(\mathbf{A}, \boldsymbol{\alpha})+\Psi(\boldsymbol{\alpha})+g(\mathbf{A})
$$

where $f$ is the loss between the mixture $\mathbf{x}$ and the synthesis coefficients $\boldsymbol{\alpha}$ of sources via the mixing matrix A. $\Psi$ models the sparsity assumption of the sources coefficients $\boldsymbol{\alpha}$. We choose here a classic $\ell_{1}$ norm for the sake of simplicity, but various other choices can be made (see for example [6] and references therein). $g(\mathbf{A})$ is mainly used to avoid the separation ambiguity [7] such as the scaling and permutation problem between $\mathbf{s}$ and $\mathbf{A}$. Using the $\ell_{2}$ loss, the problem be- 
comes:

$$
\min _{\boldsymbol{\alpha}, \mathbf{A}} L(\mathbf{A}, \boldsymbol{\alpha})=\frac{1}{2}\left\|\mathbf{x}-\mathbf{A} \boldsymbol{\alpha} \boldsymbol{\Phi}^{*}\right\|_{2}^{2}+\lambda\|\boldsymbol{\alpha}\|_{1}+\imath_{\mathcal{B}}(\mathbf{A})
$$

where $\imath_{\mathcal{B}}$ is the indicator function of a closed convex set $\mathcal{B}$ which is :

$$
\iota_{\mathcal{B}}(\mathbf{A})=\left\{\begin{array}{lc}
0 & \text { if }\left\|\mathbf{a}_{n}\right\|^{2} \leq 1 \\
+\infty & \text { otherwise }
\end{array} \forall n\right.
$$

where $\mathbf{a}_{n}$ is the $\mathrm{n}$-th column of $\mathbf{A}$.

This functional appears quite naturally in BSS as soon as one wants to exploit sparsity. More particularly, a similar functional was proposed in the so-called Generalized Morphological Component Analysis (GMCA) [8] in the overdetermined setting for images. One can notice that this kind of functional also appears in Dictionary Learning (DL) problem [9].

\subsection{GMCA-like method}

In order to minimize (3), the authors of [8] proposed an intuitive algorithm inspired by alternating minimization, followed by a projection of $\mathbf{A}$ on (4) at each step. A practical version of this algorithm (without the "morphological hypothesis" for the sake of clarity) is given in Alg. 1.

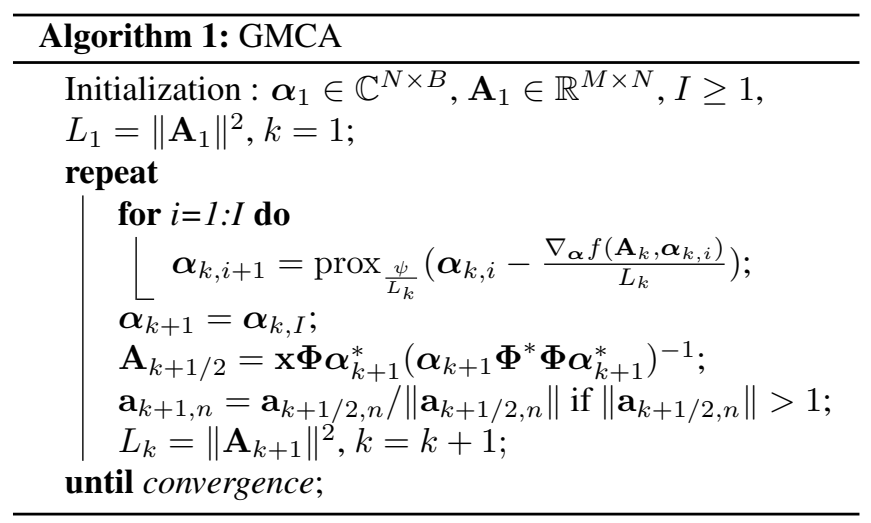

where $\nabla_{\boldsymbol{\alpha}} f\left(\mathbf{A}_{k}, \boldsymbol{\alpha}\right)=-\mathbf{A}_{k}^{T}\left(\mathbf{x}-\mathbf{A}_{k} \boldsymbol{\alpha} \boldsymbol{\Phi}^{*}\right) \boldsymbol{\Phi}$ and $\operatorname{prox}_{\frac{\psi}{L_{k}}}(\mathbf{z})=\operatorname{argmin}_{\boldsymbol{\alpha}} \frac{1}{2}\|\mathbf{z}-\boldsymbol{\alpha}\|^{2}+\frac{1}{L_{k}} \psi(\boldsymbol{\alpha})$ is the proximity operator of $\psi$, which reduces to the soft-thresholding for the $\ell_{1}$ norm [10].

However, and despite all the convergence studies of the alternating minimization approach $[11,12]$, and especially because of the projection step, the theoretical convergence of GMCA is not established. Thanks to the recent works [13,14], one can use Alg. 2 in order to minimize (3)

where $\mathbf{G}_{k}$ and $\mathbf{H}_{k}$ are some symmetric positive definite matrices, and

$$
\operatorname{prox}_{\mathbf{U}, \psi}(\mathbf{z})=\underset{\boldsymbol{\alpha}}{\operatorname{argmin}} \frac{1}{2}\langle(\mathbf{z}-\boldsymbol{\alpha}) \mathbf{U}, \mathbf{z}-\boldsymbol{\alpha}\rangle+\psi(\boldsymbol{\alpha})
$$

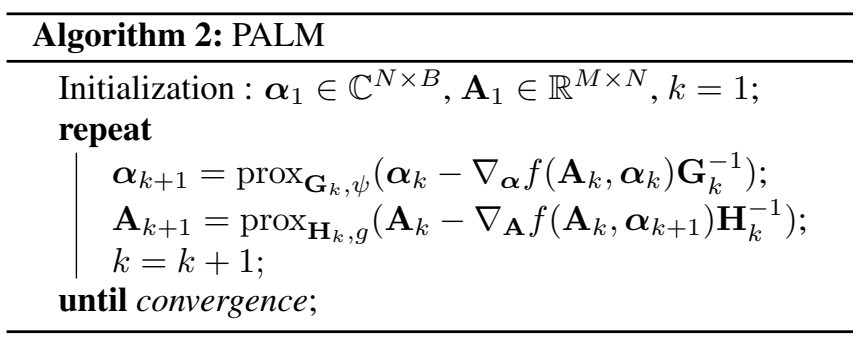

for any symmetric positive definite matrix $\mathbf{U}$.

The simplest choice $\mathbf{G}_{k}=L_{k}$ and $\mathbf{H}_{k}=\left\|\mathbf{s}_{k}\right\|^{2} \mathbf{I}$ leads to the PALM (Proximal Alternating Linearized Minimization) algorithm [13], and can be used directly with convergence guaranties. Another interesting choice is setting $\mathbf{G}_{k}=L_{k} \mathbf{I}$ and $\mathbf{H}_{k}=\frac{\partial f\left(\mathbf{A}, \boldsymbol{\alpha}_{k+1}\right)^{2}}{\partial^{2} \mathbf{A}}=\boldsymbol{\alpha}_{k+1} \boldsymbol{\Phi}^{*} \boldsymbol{\Phi} \boldsymbol{\alpha}_{k+1}^{*}$. In this case, the second step of Alg. 2 reads:

$$
\begin{aligned}
& \mathbf{A}_{k+1 / 2}=\mathbf{x} \boldsymbol{\Phi} \boldsymbol{\alpha}_{k+1}^{*}\left(\boldsymbol{\alpha}_{k+1} \boldsymbol{\Phi}^{*} \boldsymbol{\Phi} \boldsymbol{\alpha}_{k+1}^{*}\right)^{-1} \\
& \mathbf{A}_{k+1}=\operatorname{prox}_{\mathbf{H}_{k}, \imath_{\mathcal{B}}}\left(\mathbf{A}_{k+1 / 2}\right)
\end{aligned}
$$

It is clear that the first step of (5) is the least squares solution of $f(\mathbf{A})$ for $\mathbf{A}$ and the second step is a variable metric projection induced by $\boldsymbol{\alpha}_{k+1} \boldsymbol{\Phi}^{*} \boldsymbol{\Phi} \boldsymbol{\alpha}_{k+1}^{*}$. Unfortunately, this last projection can be difficult to solve. But, if at each step the estimated sources $\mathbf{s}_{k}=\boldsymbol{\alpha}_{k} \boldsymbol{\Phi}^{*}$ are decorrelated, i.e. $\mathbf{s}_{k} \mathbf{s}_{k}^{T}=\boldsymbol{\alpha}_{k} \boldsymbol{\Phi}^{*} \boldsymbol{\Phi} \boldsymbol{\alpha}_{k}^{*}=\mathbf{I}$ then it reduces to a simple orthogonal projection.

Next section proposes two approaches to take this decorrelation into account.

\section{BSS WITH SPARSITY AND DECORRELATION}

We present two approaches which consider some decorrelation on the sources. The first approach shows that this decorrelation appears in the estimation of the mixing matrix, by adding a simple regularization. The second approach introduces a projection step which ensures the decorrelation of the sources during the algorithm.

\subsection{When regularisation on $\mathrm{A}$ implies a numerical decor- relation}

Rather than (3), we propose to minimize the following functional, where a $\ell_{2}$ regularizer is added on the mixing matrix $\mathbf{A}$, and the constraint on the $\ell_{2}$ ball is replaced by the $\ell_{2}$ circle:

$$
\min _{\boldsymbol{\alpha}, \mathbf{A}} \frac{1}{2}\left\|\mathbf{x}-\mathbf{A} \boldsymbol{\alpha} \boldsymbol{\Phi}^{*}\right\|_{2}^{2}+\frac{\mu}{2}\|\mathbf{A}\|_{2}^{2}+\lambda\|\boldsymbol{\alpha}\|_{1}+\imath_{\mathcal{C}}(\mathbf{A})
$$

with:

$$
\imath_{\mathcal{C}}(\mathbf{A})=\left\{\begin{array}{lc}
0 & \text { if }\left\|\mathbf{a}_{n}\right\|^{2}=1 \\
+\infty & \text { otherwise }
\end{array} \forall n\right.
$$


Notice that this constraint is not convex, but the PALM algorithm (Alg. 2 with $\mathbf{G}_{k}=L_{k}$ and $\mathbf{H}_{k}=\left\|\mathbf{s}_{k}\right\|^{2} \mathbf{I}$ ) can still be used with the same guaranties.

Now, if one chooses $f(\mathbf{A}, \boldsymbol{\alpha})=\frac{1}{2}\left\|\mathbf{x}-\mathbf{A} \boldsymbol{\alpha} \boldsymbol{\Phi}^{*}\right\|_{2}^{2}+$ $\frac{\mu}{2}\|\mathbf{A}\|_{2}^{2}$ in Alg. 2, with the particular choice $\mathbf{G}_{k}=L_{k}$ and $\mathbf{H}_{k}=\frac{\partial f\left(\mathbf{A}, \boldsymbol{\alpha}_{k+1}\right)^{2}}{\partial^{2} \mathbf{A}}=\boldsymbol{\alpha}_{k+1} \boldsymbol{\Phi}^{*} \boldsymbol{\Phi} \boldsymbol{\alpha}_{k+1}^{*}+\mu \mathbf{I}$, the second step of Alg. 2 becomes:

$$
\begin{aligned}
& \mathbf{A}_{k+1 / 2}=\mathbf{x} \boldsymbol{\Phi} \boldsymbol{\alpha}_{k+1}^{*}\left(\boldsymbol{\alpha}_{k+1} \boldsymbol{\Phi}^{*} \boldsymbol{\Phi} \boldsymbol{\alpha}_{k+1}^{*}+\mu \mathbf{I}\right)^{-1} \\
& \mathbf{A}_{k+1} \in \operatorname{prox}_{\mathbf{H}_{k}, \imath_{\mathcal{C}}}\left(\mathbf{A}_{k+1 / 2}\right)
\end{aligned}
$$

Because of the constraint on the unit circle, the choice of the parameter $\mu$ does not change the minimizer of (6). Then, by choosing $\mu$ large enough to have $\left(\boldsymbol{\alpha}_{k+1} \boldsymbol{\Phi}^{*} \boldsymbol{\Phi} \boldsymbol{\alpha}_{k+1}^{*}+\right.$ $\mu \mathbf{I})^{-1}=\frac{1}{\mu} \mathbf{I}+\varepsilon$ where $\varepsilon$ is of order of the machine precision, the projection simply reads

$$
\begin{aligned}
& \mathbf{A}_{k+1 / 2}=\mathbf{x} \boldsymbol{\Phi} \boldsymbol{\alpha}_{k+1}^{*} / \mu \\
& \mathbf{a}_{k+1, n}=\mathbf{a}_{k+1 / 2, n} /\left\|\mathbf{a}_{k+1 / 2, n}\right\|
\end{aligned}
$$

We give in Alg. 3 this particular version of the BC-VMFB algorithm which is called Regularized-GMCA

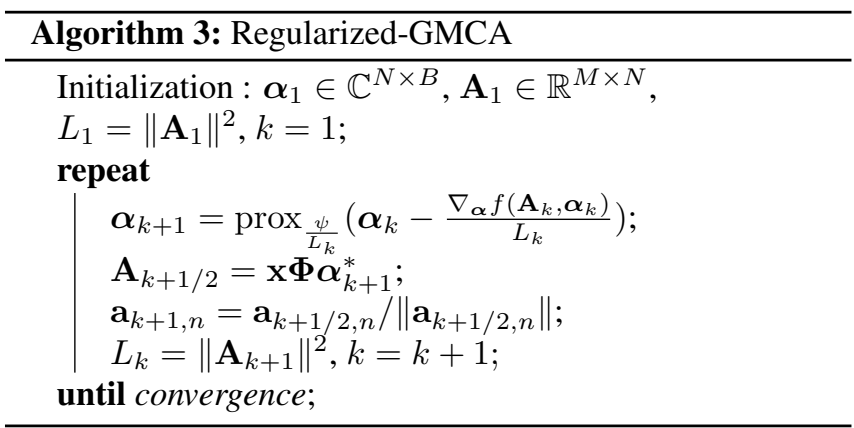

It can be proven that (9) enforces the decorrelation of the sources estimation in the over-determined noiseless case ( $\lambda \rightarrow 0$ in (3)), by considering simple optimality conditions. We omit the proof due to the lack of space, but this claim will be clearly supported by the experiments.

\subsection{A decorrelated GMCA}

The above algorithm only enforces the decorrelation in the over-determined noiseless case. So we develop here an algorithm which enforces decorrelation in all scenarios by implementing a decorrelation projection at each iteration.

We first present the following proposition for Minimized Mean-Squared Error (MMSE) decorrelation, which is a consequence of the result presented in [15]

Proposition 1. Let $\mathbf{s} \in \mathbb{R}^{N \times T}$ be a 0-mean signal matrix with a positive definite covariance matrix $\Sigma_{\mathbf{s}}=\mathbf{s s}^{T}$. Let $\mathbf{W}$ be the optimal decorrelation transformation that minimizes the Mean-Squared Error (MSE) between the input $\mathbf{s}$ and the output $\mathbf{y}=\mathbf{W} \mathbf{s}$ with covariance $\Sigma_{\mathbf{y}}$ being a diagonal matrix:

$$
\min _{\mathbf{y}=\mathbf{W} \mathbf{s}}\|\mathbf{s}-\mathbf{y}\|_{2}^{2}
$$

then $\mathbf{W}=\operatorname{diag}\left(\Sigma_{\mathbf{S}}^{1 / 2}\right) \Sigma_{\mathbf{S}}^{-1 / 2}$ where diag (.) is the diagonal matrix formed with its diagonal elements.

Proof. We consider the problem (10) in the following way:

$$
\begin{gathered}
\min _{\overline{\mathbf{W}}}\|\mathbf{s}-\overline{\mathbf{W}} \overline{\mathbf{y}}\|_{2}^{2} \\
\text { s.t. } \overline{\mathbf{W}} \overline{\mathbf{W}}^{T}=\mathbf{D}
\end{gathered}
$$

where $\overline{\mathbf{y}}$ is the whitened signal, i.e. $\overline{\mathbf{y}}=\Sigma_{\mathbf{S}}^{-1 / 2} \mathbf{S}$ and $\mathbf{D}$ is any diagonal matrix. Then the MSE can be rewritten as :

$$
e=\|\mathbf{s}\|_{2}^{2}+\|\overline{\mathbf{W}} \overline{\mathbf{y}}\|_{2}^{2}-2\langle\mathbf{s}, \overline{\mathbf{W}} \overline{\mathbf{y}}\rangle
$$

According to Cauchy-Schwarz inequality, one has $\langle\mathbf{s}, \overline{\mathbf{W}} \overline{\mathbf{y}}\rangle \leq$ $\|\mathbf{s}\|_{2}\|\overline{\mathbf{W}} \overline{\mathbf{y}}\|_{2}$ with equality if and only if $\overline{\mathbf{W}}$ is a diagonal matrix. We note $\bar{d}_{i}$ the $\mathrm{i}$-th element on the diagonal of $\mathbf{\mathbf { W }}$, then we can reformulate $e$ as :

$$
e=\sum_{i=1}^{N}\left(\mathbf{s}_{i}-\bar{d}_{i} \overline{\mathbf{y}}_{i}\right)\left(\mathbf{s}_{i}-\bar{d}_{i} \overline{\mathbf{y}}_{i}\right)^{T}
$$

where $\mathbf{s}_{i}$ is the $\mathrm{i}$-th row of $\mathbf{s}$ and $\overline{\mathbf{y}}_{i}$ is the $\mathrm{i}$-th row of $\mathbf{y}$. Now minimizing $e$ with respect to $\bar{d}_{i}$ is a simple problem with the solution $\bar{d}_{i}=\mathbf{s}_{i} \overline{\mathbf{y}}_{i}^{T}$ which means $\mathbf{W}=\operatorname{diag}\left(\mathbf{s} \overline{\mathbf{y}}^{T}\right)$.

By incorporating such a projection in the GMCA algorithm, we obtain the decorrelated-GMCA in Alg. 4.

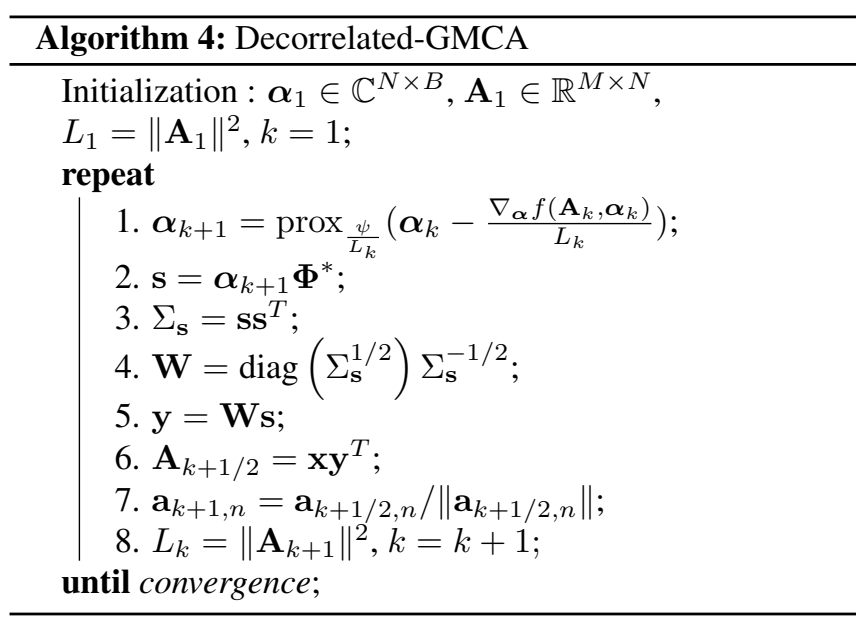

In the above algorithm, the MMSE decorrelation projection in each iteration (step 2 to 5) is designed to make sure that the estimated signals are decorrelated and to keep their energy unchanged at the same time. It is worthy mentioning that these extra steps will not bring too much computational cost. However, the convergence proof of this algorithm remains an open problem. 


\section{NUMERICAL EXPERIMENTS}

After the presentation of the experimental setup, we describe in this section the performances of the proposed methods in different scenarios.

\subsection{Experimental setup}

For all the experiments, the signals are taken from 7 different sets of male or female speech sources (available in SiSEC2011 [16]). The number of sources is set to 3 while the number of microphones varies among 2, 3 and 5. The STFT was computed with half-overlapping tight Hann window using the ltfat toolbox [17] and the performances were assessed using Signal to Distorsion Ratio (SDR) and Signal to Interference Ratio (SIR) [18]. The SDR indicates the overall quality of each estimated source compared to the target, while the SIR reveals the amount of residual crosstalk from the other sources. The robustness to the noise is also evaluated by adding a white Gaussian noise at various level of input SNR. All the algorithms are initialized randomly and the hyperparameter $\lambda$ is chosen as the one that corresponds to the best performance from various values.

\subsection{Over-determined BSS}

We present here the performances in determined $(M=3$, $N=3$ ) and over-determined ( $M=5, N=3$ ) cases. FastICA [19] is evaluated as a baseline. The performances are shown in Fig. 1.

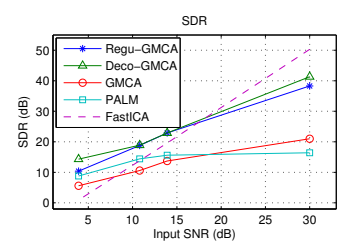

$\mathrm{SDR}$

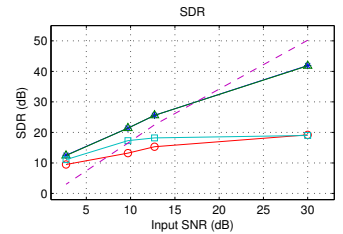

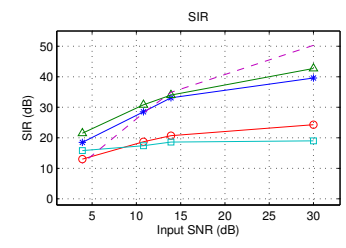

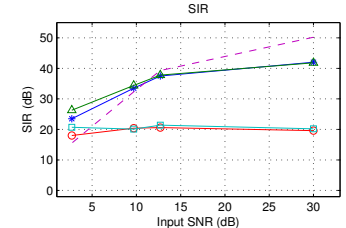

Fig. 1. Top: Performance evaluation in determined case with $M=3$ and $N=3$. Bottom: Performance evaluation in over-determined case with $M=5$ and $N=3$

Although the FastICA outperforms other algorithms when the input noise is negligeable, its performance decreases rapidly as the noise increases, and becomes outperformed by other approaches. One can remark that decorrelated-GMCA and regularized-GMCA perform similarly in over-determined case and outperform the PALM minimization of (3). This ob- servation confirms the intuition that the decorrelation can help the estimation.

\subsection{Under-determined BSS}

We evaluate the algorithms in the under-determined case ( $M=2, N=3$ ) using the mixing matrix from [16] which has a condition number equal to 4.2. For this case, we use the DUET [4] algorithm as a baseline. The SDR/SIR performances are displayed on Fig. 2
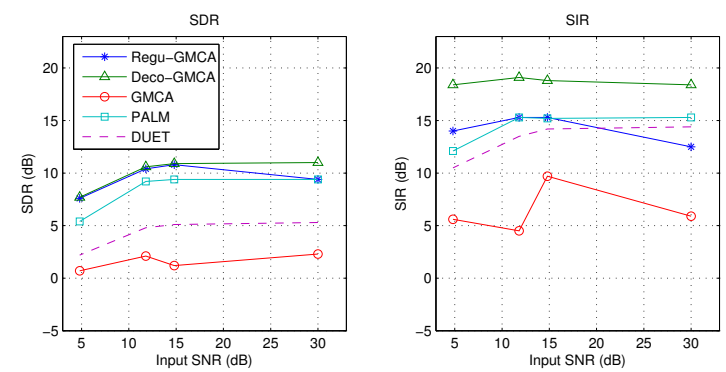

Fig. 2. Performance evaluation in under-determined case with $M=2$ and $N=3$

As expected, the decorrelated-GMCA outperforms all the other algorithms in both SDR and SIR, for every level of input noise. It is interesting to note that, for a high input SNR the PALM algorithm outperforms the regularized-GMCA in term of SIR, while their performances are similar in term of SDR. This confirms that the regularized-GMCA ensures a decorrelated solution only in the over-determined case.

\subsection{Computational comparison}

We end this experimental section by giving some indications about the computational time of different methods. Fig. 3 shows the separation performance in terms of the average computational time in the under-determined noiseless case. It can be noticed that even though decorrelated-GMCA takes
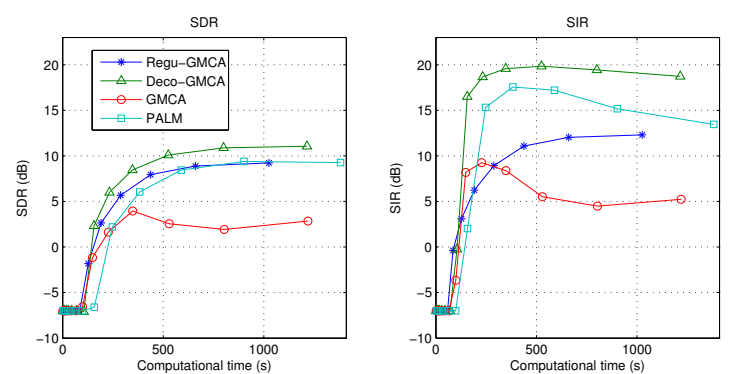

Fig. 3. Computaional time in under-determined noiseless case

more steps in each iteration, it does not cost too much computational time to reach its best performance. Besides, DUET takes less than one second to proceed the separation on a laptop with Pentium CPU (2.1 GHz), 2 Gb memory. 


\section{DISCUSSION AND CONCLUSION}

We studied and proposed several iterative methods to separate instantaneous mixture in under-determined and overdetermined BSS. In particular, we developped an approach to combine both the decorrelation in the time domain and the sparsity in the time-frequency domain of the sources. It has been shown that this approach leads to better separation in both SDR and SIR, and is very robust to the noise.

In the decorrelated-GMCA, we chose to maintain the energy of the sources, and forced each columns of the mixing matrix to have a unit norm to deal with the scaling ambiguity. In theory, it is totally possible to force the sources to have a unit norm, and then to have $\mathbf{s s}^{T}=\mathbf{I}$ to release the constraint on the mixing matrix. Surprisingly enough, this approach does not work as well as the proposed approach. We then chose to only present the decorrelated-GMCA as in this article.

Future works will focus on extending the decorrelation and sparsity combination to convolutive mixture. Structured sparsity will also be considered to take signal structures into account. From an applicative point of view, such an approach may be applied to M/EEG and fMRI signals.

\section{REFERENCES}

[1] P. Comon, "Independent component analysis, a new concept?," Signal processing, vol. 36, no. 3, pp. 287314, 1994.

[2] P. Comon and C. Jutten, Handbook of Blind Source Separation: Independent component analysis and applications, Academic press, 2010.

[3] S. Arberet, R. Gribonval, and F. Bimbot, "A robust method to count and locate audio sources in a multichannel underdetermined mixture," Signal Processing, IEEE Transactions on, vol. 58, no. 1, pp. 121-133, 2010.

[4] O. Yilmaz and S. Rickard, "Blind separation of speech mixtures via time-frequency masking," Signal Processing, IEEE transactions on, vol. 52, no. 7, pp. 1830 1847, 2004.

[5] I. Daubechies, E. Roussos, S. Takerkart, M. Benharrosh, C. Golden, K. D'ardenne, W. Richter, J.D. Cohen, and J. Haxby, "Independent component analysis for brain fmri does not select for independence," Proceedings of the National Academy of Sciences, vol. 106, no. 26, pp. 10415-10422, 2009.

[6] P. Balazs, M. Dörfler, M. Kowalski, and B. Torrésani, "Adapted and adaptive linear time-frequency representations: a synthesis point of view," Signal Processing Magazine, IEEE, vol. 30, no. 6, pp. 20-31, 2013.

[7] H. Boumaraf, Séparation aveugle de mélanges convolu- tifs de sources, Ph.D. thesis, Université Joseph-FourierGrenoble I, 2005.

[8] J. Bobin, J.-L. Starck, J. Fadili, and Y. Moudden, "Sparsity and morphological diversity in blind source separation," Image Processing, IEEE Transactions on, vol. 16, no. 11, pp. 2662-2674, 2007.

[9] J. Mairal, J. Ponce, G. Sapiro, A. Zisserman, and F. Bach, "Supervised dictionary learning," in $\mathrm{Ad}$ vances in neural information processing systems, 2009, pp. 1033-1040.

[10] P.L. Combettes and V.R. Wajs, "Signal recovery by proximal forward-backward splitting," Multiscale Modeling and Simulation, vol. 4, no. 4, pp. 1168-1200, Nov. 2005.

[11] P. Tseng, "Convergence of a block coordinate descent method for nondifferentiable minimization," Journal of optimization theory and applications, vol. 109, no. 3, pp. 475-494, 2001.

[12] W. I. Zangwill, Nonlinear programming: a unified approach, Prentice-Hall Englewood Cliffs, NJ, 1969.

[13] J. Bolte, S. Sabach, and M. Teboulle, "Proximal alternating linearized minimization for nonconvex and nonsmooth problems," Mathematical Programming, pp. 136, 2013.

[14] E. Chouzenoux, J.-C. Pesquet, and A. Repetti, "A block coordinate variable metric forward-backward algorithm," https://hal.archives-ouvertes.fr/hal-00945918/, 2013.

[15] Y. C Eldar and A. V. Oppenheim, "MMSE whitening and subspace whitening," Information Theory, IEEE Transactions on, vol. 49, no. 7, pp. 1846-1851, 2003.

[16] S. Araki, F. Nesta, E. Vincent, Z. Koldovskỳ, G. Nolte, A. Ziehe, and A. Benichoux, "The 2011 signal separation evaluation campaign (SiSEC2011):-audio source separation," in Latent Variable Analysis and Signal Separation, pp. 414-422. Springer, 2012.

[17] P. L Sondergaard, B. Torrésani, and P. Balazs, "The linear time frequency analysis toolbox," International Journal of Wavelets, Multiresolution and Information Processing, vol. 10, no. 04, 2012.

[18] E. Vincent, R. Gribonval, and C. Févotte, "Performance measurement in blind audio source separation," Audio, Speech, and Language Processing, IEEE Transactions on, vol. 14, no. 4, pp. 1462-1469, 2006.

[19] A. Cichocki, S.-I. Amari, K. Siwek, T. Tanaka, A. H. Phan, R. Zdunek, S. Cruces, P. Georgiev, Y. Washizawa, and Z. Leonowicz, "Icalab toolboxes," URL: http://www. bsp. brain. riken. jp/ICALAB, 2007. 